\title{
Comparison of in vivo biocompatibilities between parylene-C and polydimethylsiloxane for implantable microelectronic devices
}

\author{
DONG SUP LEE, SU JIN KIM, EUN BI KWON, CHEOL WHEE PARK ${ }^{\dagger}$, SU MIN JUN ${ }^{\ddagger}$, \\ BUMKYOO CHOI ${ }^{+}$and SAE WOONG KIM* \\ Department of Urology, ${ }^{\dagger}$ Division of Nephrology, Department of Internal Medicine, The Catholic University of Korea, \\ Seoul, Korea \\ ${ }^{\ddagger}$ Department of Mechanical Engineering, Sogang University, Seoul, Korea
}

MS received 5 February 2013

\begin{abstract}
Implantable devices are often composed of or coated with different biologically compatible materials based on their requirements. Selecting a surface material for an implantable device is not an easy task, and it is necessary to compare the biocompatibilities of the available surface materials. In this study, we perform a comparison of the in vivo biocompatibilities of polydimethylsiloxane (PDMS) and para-xylyene polymer (parylene-C) as they are considered to be candidates for a coating material for implantable microelectronic devices. For in vivo biocompatibility testing, fifty four male Sprague-Dawley rats were used for testing, and they were divided into three groups (PDMS, parylene-C and a positive control). At one, four and twelve weeks after implantation of the test object, the density of inflammatory cells and the granulation layer thickness were recorded for each group and compared with other groups using visible light and fluorescence microscopy. The thickness of the granulation layer tended to decrease over time for all of the experimental groups, whereas the granulation layer thickness remained constant in the positive control group. The thinnest capsular layer was observed for the parylene-C group and fewest inflammatory cells were observed in this group during the entire experimental period. Macrophage infiltration was minimal, even at one week, and was not observed thereafter. The parylene-C group showed better biocompatibility than the PDMS groups, both for acute and chronic implantation. Thus, parylene-C is the best candidate of the tested materials for applications involving permanent implantable micro-devices.
\end{abstract}

Keywords. In vivo biocompatibility; polydimethylsiloxane; parylene-C.

\section{Introduction}

Sustained high detrusor pressure often results from a poorly compliant bladder, and when left uncorrected, it further places the upper urinary tract at risk (Gerridzen et al 1992). For this reason, the management and follow up of neurogenic bladder patients with high intravesicle pressure includes regular urodynamic evaluation to assess the present condition of the urinary bladder and a recommendation of frequent emptying of the urinary bladder by clean intermittent catheterization with anticholinergics in order to lower bladder pressure and enhance bladder compliance. A continuous bladder pressure monitoring device could enhance the quality of life in neurogenic bladder patients by reducing the chance of an expensive and bothersome urodynamic study as well as by alerting the optimal time for catheterization. Scientists have made their efforts to monitor the bladder pressure in real-time by designing novel devices. Tan et al (2009) developed an implantable wireless pressure monitoring system, where a sensor packed with polydimethylsiloxane (PDMS) was attached to the inner surface of a swine's bladder. With this type of device, however, encrustation can occur. That is,

\footnotetext{
*Author for correspondence (ksw1227@ catholic.ac.kr)
}

all the biomaterials that are used will become encrusted to some extent when exposed to urine, which can be a source of morbidity, for example, as a result of urinary tract infection (Sofer and Denstedt 2000). A study aimed at finding a solution to this problem was recently reported (Majerus et al 2011), where a sensor coated with silicon (chemical formula was not exactly commented) was implanted into the submucosal layer, to avoid contact with urine. As the device is permanently embedded beneath the mucosa, other materials, such as parylene, may be considered as a potential surface material for this micro-electronic device. Because parylene$\mathrm{C}$ is one of the most widely used coating materials for implantable electronic devices (Schwarz et al 2004), PDMS has been called a primary reference material (Bélanger and Marois 2001), and has been used in various medical fields (Scott et al 1973; Amzallag and Pynson 2007). Additionally, parylene-C coatings have been demonstrated to enhance the functionality and durability of prosthetic devices (Salem et al 2009). However, it is difficult to find in vivo data for comparison of acute and chronic biocompatibilities of both the materials, and there is a lack of consensus about biocompatibilities of these materials between papers, probably due to methodological differences, which lead authors to evaluate the in vivo biocompatibilities of these noble biomaterials 
and compared them to each other according to international guideline.

\section{Materials and methods}

The research was conducted in accordance with the guidelines for the care and use of laboratory animals from the National Institutes of Health and was approved by the Catholic University Animal Ethics Committee (Catholic University Medical College CUMC-2010-0115-03).

\subsection{Preparation of coating materials}

2.1a PDMS coating: The PDMS coating was prepared by immersing a lead coin bioimplant in a PDMS mixture solution (Sylgard 184, Dow Corning, Seoul, Korea: silocon elastomer:curing agent ratio $=10: 1)$ and maintained at a constant temperature of $80^{\circ} \mathrm{C}$ for $2 \mathrm{~h}$.

\subsection{Parylene-C coating}

At first, Parylene C Dimer (di-chloro-di-para-xylylene) was vaporized at atmospheric pressure (1 Torr) and $150{ }^{\circ} \mathrm{C}$ where the parylene was in its dimer form. Then, the material was pyrolyzed at 0.5 Torr and $690{ }^{\circ} \mathrm{C}$, where parylene was converted into the monomer para-xylene. The paraxylene is transformed into poly-para-xylene at a pressure of $0.1 \mathrm{~atm}$ and $35^{\circ} \mathrm{C}$. This resulting polymer was used to coat the lead coin bioimplant. The coating reaction was carried out using a Nuricell Parylene coating system (NPCR400, Nuricell, Seoul, Korea). The growth rate was $2 \mu \mathrm{m} / \mathrm{h}$, and the coating thickness of parylene was expected to be 4-6 $\mu \mathrm{m}$, methodologically similar to previous study (Shin et al 2003).

\subsection{In vivo biocompatibility testing}

2.3a Implantation of coating materials in rat dorsal subcutaneous tissue: Fifty four male Sprague-Dawley rats weighing 250-300 $\mathrm{g}$ and housed in a temperature- and humidity-controlled environment on a 12/12 h light-dark cycle were randomly and equally assigned to three groups by implant material. All the rats were allowed unrestricted access to standard rat food and water and were housed in the laboratory environment for at least one week before surgery. The rats were anesthetized by an intramuscular injection of ketamine (Ketamine, $75 \mathrm{mg} / \mathrm{kg}$; Yoohan Yanghang, Seoul, Korea) and xylazine (Rompun, $15 \mathrm{mg} / \mathrm{kg}$; Bayer Korea, Seoul, Korea) for surgical manipulation. The back area of each rat was sterilized with a $10 \%$ povidone-iodine solution and incised using sterile techniques. The subcutaneous tissues were exposed and a lead coin with or without a coating was placed in direct contact with the exposed tissue, consistent with ISO 10993-6. Eighteen rats (group 1) were implanted with lead coins coated with PDMS and another eighteen rats (group 2) with lead coins coated with paryleneC. The other rats (group 3) were implanted with uncoated lead coins (for use as a positive control group). Six rats in each study group were randomly sacrificed at one, four, and twelve weeks post-surgery to evaluate biocompatibility. Nine sections were randomly obtained for histological evaluation in each group.

\subsection{Visible light microscopy assessment of tissue reaction}

Tissue from around the implanted material was collected and fixed in $10 \%$ neutral formalin for one day. Fixed tissues were prepared as samples for visible light microscopy by dehydrating with alcohol; embedding the tissue in paraffin; sectioning $(5 \mu \mathrm{m})$ using a microtome; and staining with hematoxylin and eosin (H\&E) and Masson's trichrome. A pathologist who was unaware of the experimental protocols examined the tissues taken from the implantation site using a visible light microscope and measured the maximum thickness of the granulation layer and inflammatory layer adjacent to the implantation site. The widest area was used as the measurement of the granulation layer thickness, and the most concentrated area was used to determine the inflammatory reaction. From this information, density of the inflammatory cells (macrophage and neutrophil) within a $100 \times 100 \mu \mathrm{m}$ area was recorded, similar to previous reports (Bélanger and Marois 2001; Wang et al 2010). We then divided the tissue into groups based on relative inflammatory degrees: minimal ( $<25$ inflammatory cells), mild ( $<50$ inflammatory cells), moderate ( $<75$ inflammatory cells) and severe $(>75$ inflammatory cells) according to the mean value of the cell density, based on the methodology from a previous report (Wang et al 2010).

\subsection{Fluorescence microscopy assessment of inflammatory reactions}

Sections of tissue were immobilized on slides, washed with $100 \%$ xylene for $3 \mathrm{~min}$ each, immersed in ethanol from $100 \%$ to $60 \%$ sequentially for $2 \mathrm{~min}$ each, and then washed with ethanol for $5 \mathrm{~min}$. The slides were added to a $0.01 \%$ sodium citrate solution ( $\mathrm{pH} \mathrm{6}$ ), boiled in a microwave oven and cooled for $20 \mathrm{~min}$ at room temperature. To inhibit nonspecific reactions during staining, the samples were reacted with a blocking solution ( $1.5 \%$ normal goat serum, $1.5 \%$ normal horse serum, $1 \%$ bovine serum albumin and $0.1 \%$ Triton $\mathrm{X}-100$ in phosphate buffered saline, PBS) at room temperature for $1 \mathrm{~h}$ and reacted with fluorescein isothiocyanate (FITC)-conjugated MAC387 macrophage primary antibody (Abcam, Cambridge, MA, USA) at room temperature for $2 \mathrm{~h}$. The samples were then washed with PBS three times and reacted at room temperature for $1 \mathrm{~h}$ with secondary antibody (Alexa Fluor 568 goat anti-rabbit IgG; Invitrogen, Carlsbad, CA, USA) diluted to 1:500 with blocking solution. The samples were then washed with PBS three times, mounted with 
4',6-diamidino-2-phenylindole (DAPI) and examined using a fluorescence microscope.

\subsection{Data analyses}

Statistical analysis was performed using SPSS software version 15.0 (SPSS, Chicago, IL, USA). The results were assessed by using the mean values \pm the standard deviations. Comparisons between the groups at one, four, and twelve weeks during the whole experimental period were performed using the Mann-Whitney U test, whereas comparison within each group was done using the Friedman test. Graphics were created using Prism software version 5.03 (GraphPad Software, San Diego, CA, USA).

\section{Results and discussion}

A visible-light microscopic examination with the H\&E-stain under a low-power field $(40 \times)$ revealed that the implantation site was distinguishable from normal subcutaneous tissue by a granulation capsule with or without a central cleft (see figure 1). Inflammatory cells were concentrated in the centre of the capsule in which the foreign body reaction was taking place. In the PDMS group, it was not easy to find the granulation layers in the low-power field after the 12-week period. In the parylene- $C$ group, the granulation layer was very thin, even after the one-week period, and we could not find the layer in any samples after the four-week period. In the positive control group, the layer was identified only after the 12-week period.

These layers were more easily identified when Masson's trichrome stain was used (see figure 2A). Closer observations using Masson's trichrome stain revealed that the thickness of the layer tended to decrease with time in groups 1 and 2 , whereas the thickness remained constant in group 3 . The mean thicknesses of the granulation layer in group 1 were $0.790 \pm 0.047,0.698 \pm 0.055$ and $0.317 \pm 0.045 \mathrm{~mm}$ at weeks 1, 4 and 12, respectively. The mean thicknesses of the granulation layer in group 2 were $0.570 \pm 0.065,0.278 \pm$
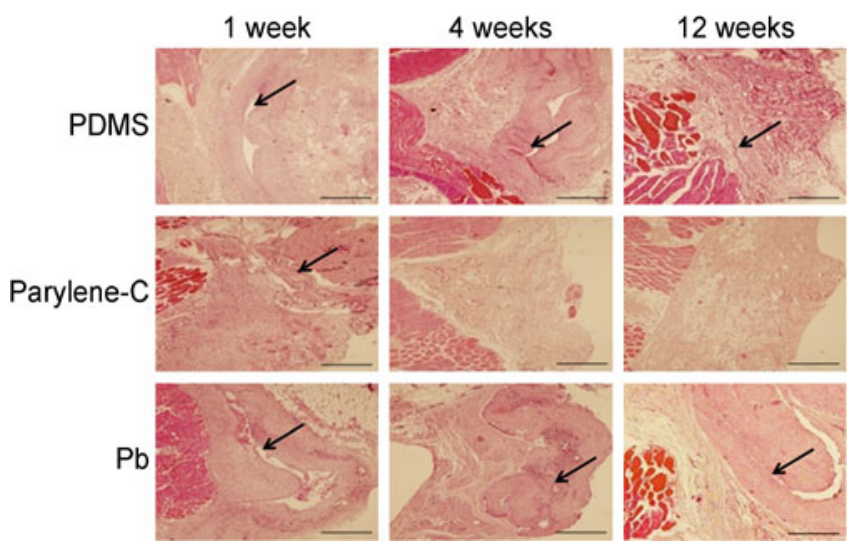

Figure 1. Histological overview with light microscopy (H\&E staining $40 \times$, arrow: implantation site, scale bar: $1.0 \mathrm{~mm}$ ).
0.076 and $0.129 \pm 0.032 \mathrm{~mm}$ at one, four and twelve weeks, respectively. Here, the granulation layer for group 2 was thinner than that for group 1 during the experimental period $(p<0 \cdot 01)$. The thickness of the layer was unchanged in group 3 during the experimental period ( $p=0.121$ ) (see figure $2 \mathrm{~B}$ ).

The presence of the inflammatory cells tended to decrease over time, and the cells were marginally distributed (see figure 3A). After one week, the mean number of inflammatory cells per $100 \times 100 \mu \mathrm{m}^{2}$ in groups 1,2 and 3 were $77.67 \pm 12.39,38.56 \pm 5.20$ and $116.0 \pm 13.98$, respectively. After four weeks, the mean numbers of inflammatory cells in groups 1, 2 and 3 were $42 \cdot 89 \pm 5 \cdot 89,22.67 \pm 2.73$ and $91.44 \pm 10 \cdot 35$, respectively. After 12 weeks, the mean numbers of inflammatory cells in groups 1,2 and 3 were $34.78 \pm 5 \cdot 31,14.0 \pm 3 \cdot 32$ and $82.56 \pm 9 \cdot 14$, respectively. Inflammatory cells in group 1 during the experimental period were more densely populated than those in group $2(p<0 \cdot 01)$ (see figure $3 \mathrm{~B}$ ). By relating this to the previously defined metrics for the degree of relative inflammation, group 1 showed severe inflammation at one week post-implantation and mild inflammation thereafter. Group 2 showed only mild inflammation at one week post-implantation and only minimal inflammation thereafter. Group 3 (the positive control) showed severe inflammation for all examinations.

For the MAC387 stain examined with fluorescence microscopy, the immune reaction from the macrophage infiltration gradually decreased and was barely evident at

A
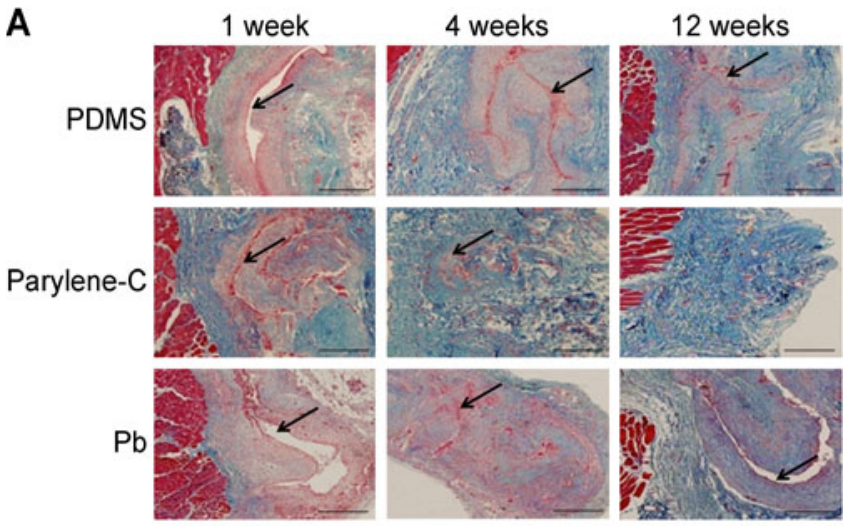

B

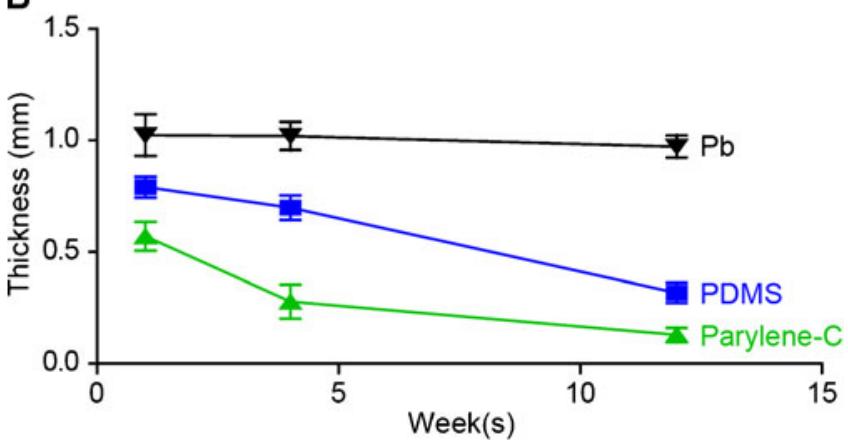

Figure 2. Thicknesses of granulation layer (Masson trichrome staining $40 \times$, arrow: implantation site, scale bar: $1.0 \mathrm{~mm}$ ). 
A

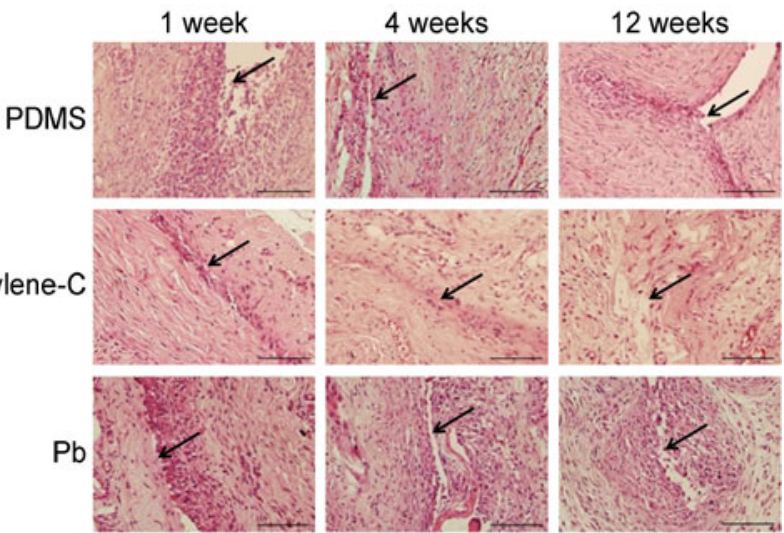

B

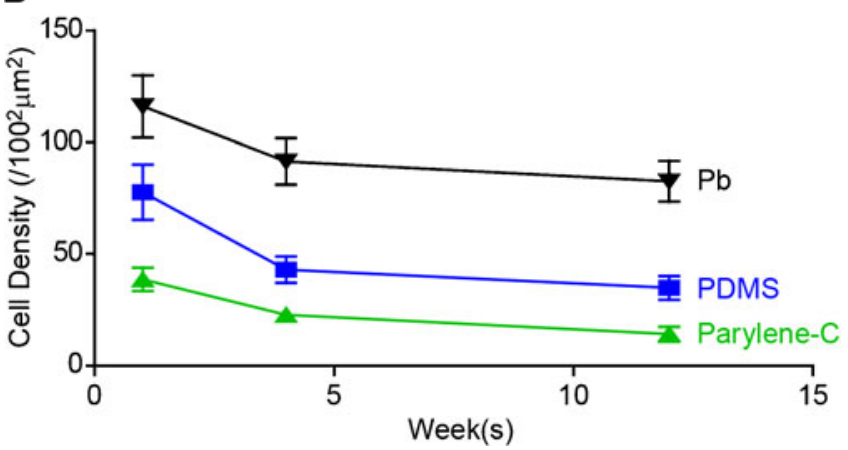

Figure 3. Inflammatory cell densities (H\&E staining $400 \times$, scale bar: $100 \mu \mathrm{m})$.

12 weeks post-implantation in groups 1 and 2 . However, in the parylene- $\mathrm{C}$ group, the macrophages were barely visible even at one week post-implantation and had nearly disappeared after four weeks. The percentages of MAC positive immune-reactive cells were more widely distributed in group 1 than in group 2 (see figure 4(A and B)).

Various micro-devices in a number of different medical fields are currently under development (Proceedings in Vienna 2010). In the case of devices used for monitoring urinary bladder pressure, embedding a pressure sensor permanently into the bladder wall to prevent encrustation must be justified with tissue compatibility testing by comparing with several available materials. Previous experimental models to monitor urinary bladder pressure have been designed using silicon-based polymers as surface materials without any comparison for biocompatibility with other materials, such as parylene-C.

Concurrently, a number of studies have introduced electronic micro-devices with parylene-based surface material (Hsu et al 2009; Hassler et al 2010). Those studies were designed with the rationale that parylene possesses good biocompatibility, excellent chemical inertia and extremely high dielectric strength compared with other widely used microfabrication materials (Feili et al 2005; Wright et al 2008). Yu et al (2009) supported the tissue compatibility of parylene as a coating material for a microelectronic device implanted in the subretinal space of rabbits but did not compare it
A
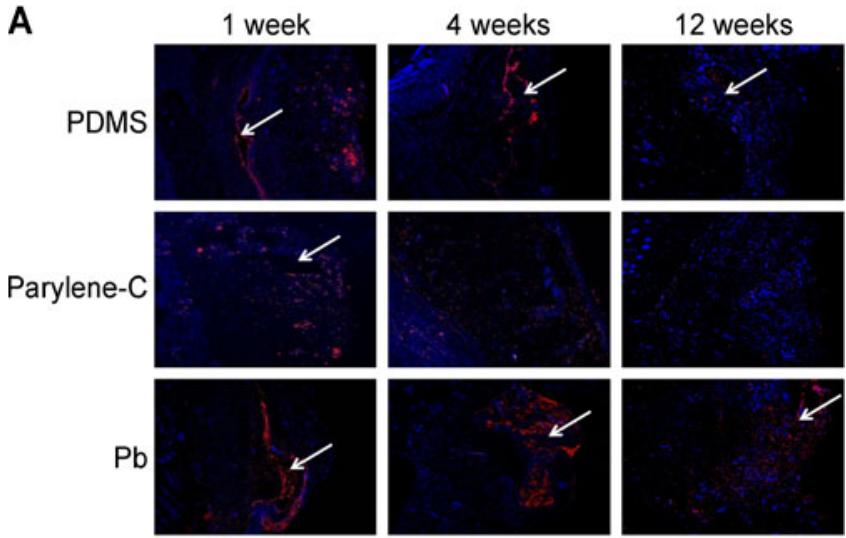

B

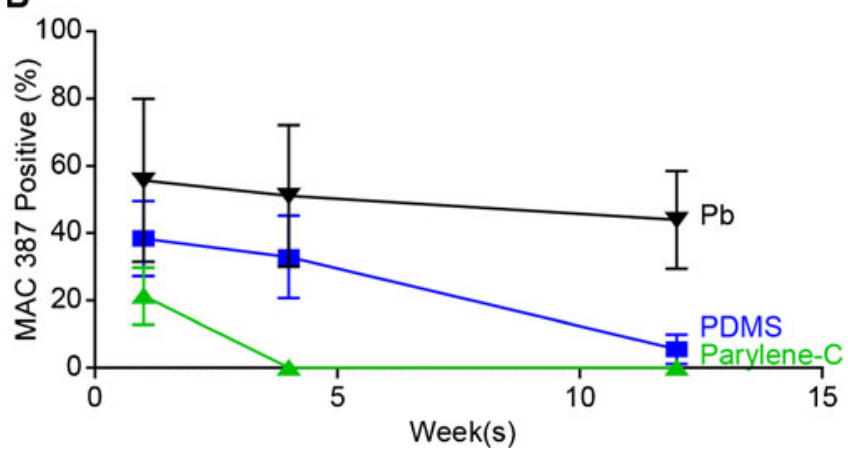

Figure 4. Macrophage infiltration around implantation site (MAC387 staining 40×, blue: cell nucleus (DAPI), red: macrophage).

with other polymers. Montezuma et al (2006) compared the biocompatibility of parylene with those of amorphous aluminum oxide, amorphous carbon, poly-vinyl-pyrrolidone and poly-ethylene-glycol in the pig retina, and they concluded that parylene was one of the most suitable coatings to microelectronic device.

However, Winslow et al (2010) in their in vivo biocompatibility study with neuronal tissue, did not find evidence that differences in the surface chemistry of implanted microelectrode arrays, whether they were coated with parylene$\mathrm{C}$ or not (uncoated planar silicon), can significantly change the foreign body response. In addition, in evaluating the surface properties of parylene-C in comparison with PDMS with respect to in vitro biocompatibility, the cell adhesion and morphology on parylene-C and PDMS were not found to be significantly different, despite a clear difference in surface roughness between the two materials, where paryleneC substrates are relatively rough $(>20 \mathrm{~nm})$ and hydrophobic (Chang et al 2007).

The medical application of a surface material for an implantable device is use-specific and is determined not only by safety itself but also by the mechanical or biochemical properties associated with the circumstances of implantation (Green et al 2008). Therefore, performing only a biological comparison of several materials may not provide enough information to make a decision about the selection of one 
of these materials as a surface material for an implantable device. Furthermore, there are many biocompatible coating materials and many newly developed techniques available for surface modification. For these reasons, this type of work, which compares in vivo biocompatibility among these two hydrophobic materials-PDMS and parylene, does not provide a standard with which to choose packing materials, but it offers insight into the micro-morphological features of these biomaterials after implantation and could be used as a reference for other research because in vivo comparisons of the biocompatibility of these materials are still lacking.

Based on the concept of biocompatibility, we compared the in vivo tissue reactions to PDMS and parylene-C. To this end, we used uncoated lead coins as a positive control group, whereas lead coins were coated with PDMS or parylene-C in experimental groups. Because lead is one of the most toxic material (Flora et al 2012), we used it instead of other metallic substrates. The lead-induced inflammatory response has been well described in a previous study (Liu et al 2012).

Generally, an acute inflammatory response lasts for a relatively short duration (a few days) (Anderson 2001). Persistent inflammatory stimuli, such as the continual presence of a foreign object, leads to chronic inflammation. To evaluate chronic inflammation, an observation period $>12$ weeks is recommended by ISO10993-6. Therefore, we started our evaluation for the immune response with one week as the turning point and set the end at 12 weeks. Dalu et al (2000) interpreted the inflammatory reaction by measuring the granulation layer thickness around the implantation site and the relative number of inflammatory cells in the layer, which is also recommended in ISO 10993-6. The infiltration of inflammatory cells adjacent to the implantation site was assessed in a previous study and that study showed that the density of the inflammatory cells after PDMS implantation peaked at two weeks and stabilized over time before reaching a nadir point at approximately six weeks (Bélanger and Marois 2001).

In our study, inflammatory cell infiltration and granulation thickness peaked at one week for all the experimental groups. The markedly decreased granulation layers and cellular densities suggested that these materials were biocompatible. The importance of these inflammatory reaction lies in that it can affect crucial functionality of micro-electronic sensor (Tan et al 2011).

Parylene-C presented excellent biocompatibility when compared with PDMS, showing that its degree of inflammation was lower and its granulation thickness was thinner than those in PDMS. In addition, the cell density and granulation thickness were significantly decreased at 12 weeks postimplantation, indicating that there were no ongoing inflammatory processes. These phenomena are more clearly seen in figure 4. Generally, monocytes are recruited to the site and differentiate into macrophages during the acute phase of inflammation. In chronic inflammation, macrophages with monocytes and lymphocytes are the main components that maintain the inflammatory process. Thus, the macrophage is the most important cell in the inflammatory reaction.
For this reason, many studies employ immunohistological stains such as MAC387, ED1 or CD4 as macrophage markers (Kim et al 2009). We used the MAC387 stain to demonstrate that the macrophage reaction was rapid; evidence of the reaction was observed after one week, and then the reaction gradually decreased thereafter. For PDMS, the immune reaction with macrophage infiltration gradually decreased and an immune reaction was barely evident at 12 weeks post-implantation. However, for the parylene-C group, the macrophages had nearly disappeared after four weeks. Comparing groups 1, 2 and 3, the inflammatory reaction associated with macrophage infiltration was lowest in the parylene-C group. As a result, the degree of inflammatory response was not constant throughout the experiment but rather decreased over time. The parylene-C group demonstrated the better biocompatibility, similar to the results obtained by visible light microscopy.

\section{Conclusions}

Parylene-C showed a minimal inflammatory reaction in the acute and chronic phases, and it showed better biocompatibility than PDMS. Parylene-C is recommended as a surface biomaterial for use with implantable electronic devices if the implantation environment is not a consideration.

\section{Acknowledgements}

This study was supported by the Korean Health Technology R\&D Project, Ministry of Health \& Welfare, Republic of Korea (A111055).

\section{References}

Amzallag T and Pynson J 2007 J. Fr. Ophthalmol. 30757

Anderson J M 2001 Annu. Rev. Mater. Res. 3181

Bélanger M C and Marois Y 2001 J. Biomed. Mater. Res. 58 467

Chang T Y, Yadav V G, De Leo S, Mohedas A, Rajalingam B, Chen C L, Selvarasah S, Dokmeci M R and Khademhosseini A 2007 Langmuir 2311718

Dalu A, Blaydes B S, Lomax L G and Delclos K B 2000 Biomaterials 211947

Feili D, Schuettler M, Doerge T, Kammer S and Stieglitz T 2005 Sens. Actuators. A Phys. 120101

Flora G, Gupta D and Tiwari A 2012 Interdiscip. Toxicol. 547

Gerridzen R G, Thijssen A M and Dehoux E 1992 J. Urol. 147 416

Green R A, Lovell N H, Wallace G G and Poole-Warren L A 2008 Biomaterials 293393

Hassler C, von Metzen R P, Ruther P and Stieglitz T 2010 J. Biomed. Mater. Res. B Appl. Biomater. 93266

Hsu J M, Rieth L, Normann R A, Tathireddy P and Solzbacher F 2009 IEEE Trans. Biomed. Eng. 5623

Kim B S, Oh J M, Kim K S, Seo K S, Cho J S, Khang G, Lee H B, Park K and Kim M S 2009 Biomaterials 30902 
Liu C M, Sun Y Z, Sun J M, Ma J Q and Cheng C 2012 Biochim. Biophys. Acta 18201693

Majerus S J A, Fletter P C, Damaser M S and Garverick S L 2011 IEEE Trans. Biomed. Eng. 58763

Montezuma S R, Loewenstein J, Scholz C and Rizzo J F 3rd 2006 Invest. Ophthalmol. Vis. Sci. 473514

Proceedings of the 10th Vienna International Workshop on Functional Electrical Stimulation and 15th IFESS Annual Conference, 2010, ISBN 978-3-900928-09-4

Salem E A, Wilson S K, Neeb A, Delk J R and Cleves M A 2009 J. Sex. Med. 62615

Schwarz J A, Contescu C I and Putyera K 2004 Dekker encyclopedia of nanoscience and nanotechnology (New York, USA: CRC press) Vol. 1, p. 263

Scott F B, Bradley W E and Timm G W 1973 Urology 1252
Shin Y S, Cho K, Lim S H, Chung S, Park S J and Chung C 2003 J. Micromech. Microeng. 13768

Sofer M and Denstedt J D 2000 Curr. Opin. Urol. 10563

Tan R et al 2009 Biomed. Microdevices 11259

Tan E L, DeRouin A J, Pereles B D and Ong K G 2011 Biosensors 1134

Wang Q, McGoron A J, Pinchuk L and Schoephoerster R T 2010 J. Biomed. Mater. Res. A93 442

Winslow B D, Christensen M B, Yang W K, Solzbacher F and Tresco P A 2010 Biomaterials 319163

Wright D, Rajalingam B, Karp J M, Selvarasah S, Ling Y, Yeh J, Langer R, Dokmeci M R and Khademhosseini A 2008 J. Biomed. Mater. Res. A85 530

Yu W, Wang X, Zhao C, Yang Z, Dai R and Dong F 2009 J. Ocul. Biol. Dis. Infor. 233 